Comparative growth rate of bottlenose dolphins

(Tursiops truncatus) from three different localities

\title{
Comparación del ritmo de crecimiento de las toninas (Tursiops truncatus) pertenecientes a tres localidades distintas
}

\author{
Arturo Romero Tenorio ${ }^{1^{*}}$ y Alberto Delgado Estrella ${ }^{2}$ \\ 'Retorno Oxpee Lote 17 casa 17, Fraccionamiento Riviera Loltún Solidaridad, 77711, Quintana Roo, México. E-mail: arturomerot@ \\ gmail.com \\ ${ }^{2}$ Facultad de Ciencias Naturales, UNACAR. Calle 56 № 4, Esq. Av. Concordia, Col. Benito Juárez, 24180, Cd. del Carmen, Campeche. \\ E-mail: delgadoestrella@gmail.com \\ ${ }^{*}$ Corresponding author
}

\begin{abstract}
Introduction: In order to know the growing rate of the captive bottlenose dolphins (Tursiops truncatus) of Via Delphi, the total length and age from three different geographic zone dolphins, from Quintana Roo and Tabasco in Mexico, and some individuals from Cuba (some dolphins were born in captivity) were recorded. The aim of this study was to establish if there were statistic differences between gender and among dolphins from different localities.

Methods: The age of 43 dolphins was determined through the analysis of the growth layers groups (GLG's), The gender of these dolphins was known, the total length of all of them, and the skull length of 12 toninas. The total sample was composed of 17 males and 26 females.

Results: Of these 43 dolphins, 13 were calves between five years to 10 months old. Sexually mature dolphins from Quintana Roo, three males of 18 years old, presented a total length mean of $=253.33 \mathrm{~cm} \pm 14 \mathrm{~cm} \mathrm{~S}$. D. (range $239-267 \mathrm{~cm}, n=3$ ). Females older than 10 years old presented a total length mean of $=250.6 \mathrm{~cm} \pm 7.5 \mathrm{~cm} \mathrm{~S}$. D. (range 239-261 cm, $n=8$ ). From de State of Tabasco, males older than nine years old, had a mean total length = $236.13 \mathrm{~cm} \pm 5.2 \mathrm{~cm} \mathrm{~S}$. D. (range $238-240 \mathrm{~cm}, n=4$ ). Females were older than eight years old, their mean total length $=239.4 \mathrm{~cm} \pm 7.4 \mathrm{~cm} \mathrm{~S}$. D. (range $227-246 \mathrm{~cm}, n=5$ ). About the Cuban dolphins, The sample from the Cuban dolphins was small (seven dolphins), they compared with dolphins of same sex and age of the two localities.
\end{abstract}

Discussion : The values of Lmax of bottlenose dolphins in the Southern of the Gulf of Mexico are similar to the bottlenose dolphins in Texas and Florida, no significant statistical differences were found. Differences in growing rates were not found in the Lmax on adult dolphins between localities nor sexes.

Key words: Age; bottlenose dolphins; Cuba; Mexico; total length.

\section{Introducción}

A fin de conocer la dinámica de cualquier población, ya sea en vida libre o en cautiverio, es necesario advertir algunos parámetros sobre su historia de vida, además su relación con el entorno. Uno de estos parámetros es la edad. Gracias a la edad en que los individuos alcanzan la madurez sexual, física y/o social, podemos saber cómo está estructurada la población. Esta información puede utilizarse junto con los datos derivados de las tasas de natalidad y mortalidad, con la finalidad de evaluar la condición actual de la población y poder predecir sus tasas de crecimiento. Estas evaluaciones 
resultan valiosas en lo referente a los delfines, sobre todo en poblaciones afectadas por diversas actividades humanas, como la caza dirigida, la alteración de su hábitat y, en el caso de los delfines, cuando son víctimas directa o indirectamente de la pesca (Myrick 1991).

La descripción de las características de la historia de vida de los miembros de una población (cetáceos por ejemplo) nos permite cuantificar los factores que influyen o controlan su tamaño y sus patrones de cambio. De tal modo, las poblaciones pueden caracterizarse por su tamaño, el que fluctúa con el tiempo (Wade 2002; Berta et al. 2006). De esta manera, la investigación, determinación y documentación de las tasas de crecimiento de los delfines son esenciales para conocer la biología de la población y establecer planes de manejo y de conservación adecuados para el bienestar de los organismos (Berta et al. 2006).

En el caso particular de las toninas (Tursiops truncatus) aunque se distribuyen mundialmente en aguas tropicales y templadas y son frecuentemente exhibidas y estudiadas en cautiverio, poco se sabe de su historia natural. Sólo se han examinado algunas poblaciones (Mead y Potter 1990).

Las toninas son los cetáceos mejor conocidos y analizados mundialmente. Se han realizado trabajos sobre estimaciones poblacionales, patrones de residencia y uso de hábitat. Incluso se han tratado cuestiones muy finas de biología molecular y genética, que nos permiten conocer la diversidad dentro de las poblaciones, los procesos evolutivos, las relaciones con otras especies, la diferenciación entre formas geográficas, así como separar, si es necesario, a individuos que se encontraban en un mismo taxón en dos o más diferentes. Hay trabajos sobre la historia natural de las toninas (Hohn 1980; Cockroft y Ross 1989; Mead y Potter 1990; Fernández y Hohn 1998), donde se describen las relaciones entre la edad, el sexo, la madurez y la reproducción en zonas geográficas bien definidas. También existen diversos trabajos cuya intención fue verificar si las poblaciones de toninas y otros cetáceos, se ven afectadas (y en qué grado) por las diversas actividades humanas (turismo, pesca, e industria productora de desechos, entre otras) (Reeves et al. 2003). En México, las toninas también son uno de los cetáceos más estudiados. Contamos con varios trabajos sobre estimaciones poblacionales, patrones de residencia, movimientos y uso de hábitat (Ballance 1990; Bazúa-Durán y Delgado-Estrella 2014), sobre la interacción de las toninas con otros delfines (Delgado-Estrella 1997) y con otros cetáceos (Gallo-Reynoso 1984). También hay estudios sobre su comportamiento (Bazúa-Durán y Delgado-Estrella 2014). Sin embargo, no encontramos ninguno sobre la historia de vida de toninas en libertad ni en cautiverio. Por ello, es importante presentar los primeros datos sobre relaciones de edad, longitud total y longitud condilobasal de toninas provenientes de los estados de Tabasco y Quintana Roo, así como el análisis de una pequeña muestra de toninas cubanas. Sin duda, al obtener estos resultados y al relacionarlos con otros estudios de conducta, ecología, biología molecular, genética, se podrá contar con un panorama más claro del estado de las poblaciones de toninas en nuestro país y tener las bases necesarias para establecer programas de manejo y conservación.

El objetivo del presente estudios es determinar los patrones de crecimiento de los individuos de tres localidades diferentes, a fin de obtener los valores de edad, longitud total y longitud condilobasal de las toninas resguardadas por Grupo Via Delphi, así como de los individuos encontrados en algunos varamientos en las costas del Golfo de México.

Para esto se determinaron las tasas de crecimiento de las toninas en cautiverio. Se comparó el ritmo de crecimiento entre los sexos y entre las diferentes localidades. Se elaboraron las curvas de crecimiento por sexo y localidad. Se analizaron las diferencias del ritmo de crecimiento de las toninas nacidas en cautiverio. Finalmente, se estableció el tipo de relación entre la edad y la longitud total de los cráneos de tonina, así como la curva de crecimiento que describa el incremento de talla de las toninas. 


\section{Materiales y métodos}

Los parques Xcaret y Xel-Ha en Quintana Roo, México, mantienen toninas en cautiverio bajo su resguardo. Estas toninas provienen de dos estados de la república mexicana (Tabasco y Quintana Roo, 42 en total). Algunos ejemplares fueron importados de Cuba (13 en total). Las mediciones corporales de las toninas se realizaron mensualmente; se tomaron en línea recta por la parte ventral de los delfines, desde la punta del rostro hasta la escotadura o muesca que divide los lóbulos caudales. Se utilizaron cintas métricas plásticas flexibles con graduación en $\mathrm{mm}$. En el caso de las crías recién nacidas, ocho de ellas de progenitores de Holbox y cuatro más de progenitores de Tabasco, se comenzaron a medir hasta que cumplieron medio año de vida aproximadamente. En etapas anteriores al medio año, las mediciones se llevaron a cabo por medio de toma de video de las crías que nadaban al lado de sus madres. Dado que se conocía la longitud total de la madre, se pudo calcular el largo total de las crías al comparar proporcionalmente a ambos individuos en las imágenes de video. Cuando hubo necesidad de manejar a las crías para su evaluación médica, se aprovechó para medirlas y se compararon estas medidas con las obtenidas en los videos. Los delfines mayores al medio año de edad fueron entrenados, para medirlos, como se señaló anteriormente. Se procuró siempre se procuró que fuera el mismo medidor con el fin de homogenizar el error.

Por otra parte, con la intención de determinar la de edad de las toninas, se extrajo un diente de cada uno de los delfines (42 mexicanos y 13 cubanos). El propósito fue analizar las capas de crecimiento de dentina y cemento. Dicho procedimiento se llevó a cabo devastando los dientes en sentido longitudinal con lijas de agua de distintos grosores hasta obtener laminillas de $800 \mu$ a $500 \mu$ de grosor, que fueron analizadas en microscopio. En la figura 3, pueden apreciarse las capas de crecimiento recién mencionadas. Básicamente se observa una secuencia de bandas longitudinales claras y oscuras que se repiten en parejas y rellenan el diente de afuera hacia adentro. En el caso de las toninas, cada par de bandas corresponde a un año de vida. Se realizó el conteo por dos y hasta por tres personas para asegurar un mejor resultado (Figura 1).

Una vez obtenida la longitud total y las edades de los delfines, se realizaron las regresiones lineales necesarias para obtener las variables ( $a$ y b) que permitieron aplicar el modelo de crecimiento correspondiente. De esta forma, se construyeron las curvas de crecimiento que describen el comportamiento de los datos para cada caso. Primero se trazó una curva con los valores de todos los delfines mexicanos y otra para los ejemplares cubanos. De estos últimos, sólo se tienen siete valores; en consecuencia, no fue posible profundizar en su análisis y solamente se compararon con los de animales mexicanos de su misma edad, longitud total y sexo.

Posteriormente, se elaboraron las curvas de crecimiento por sexo y localidad para compararlas entre sí. El modelo de crecimiento utilizado fue el de von Bertalannfy ( $\left.L=A-(A-B) e^{-(k t)}\right)$, ya que se ajustó bien a los datos y se ha comprobado su utilidad en otros estudios de mamíferos acuáticos, incluidos los de las toninas (Read et al. 1993; Fernández y Hohn 1998). Se obtuvieron los promedios de la longitud total de las toninas por localidad y sexo para compararlos entre sí; se sacó el promedio de la longitud total de las crías al nacer por sexo y localidad.

Para confirmar que la relación entre edad y longitud total de los delfines era significativa, se aplicó una prueba de " $\mathrm{t}$ ". El objetivo consistió en evaluar dicha relación y se encontró que en todos los casos la relación fue significativa.

De los decesos en cautiverio y de los restos recuperados en vida libre en las costas del sur del Golfo de México, se obtuvieron medidas craneales de estas toninas. La medición de los cráneos se realizó tomando como puntos de referencia los cóndilos occipitales y la punta del rostro (premaxila), sin contar el dentario, siempre en línea recta entre esos puntos. Para realizar lo 


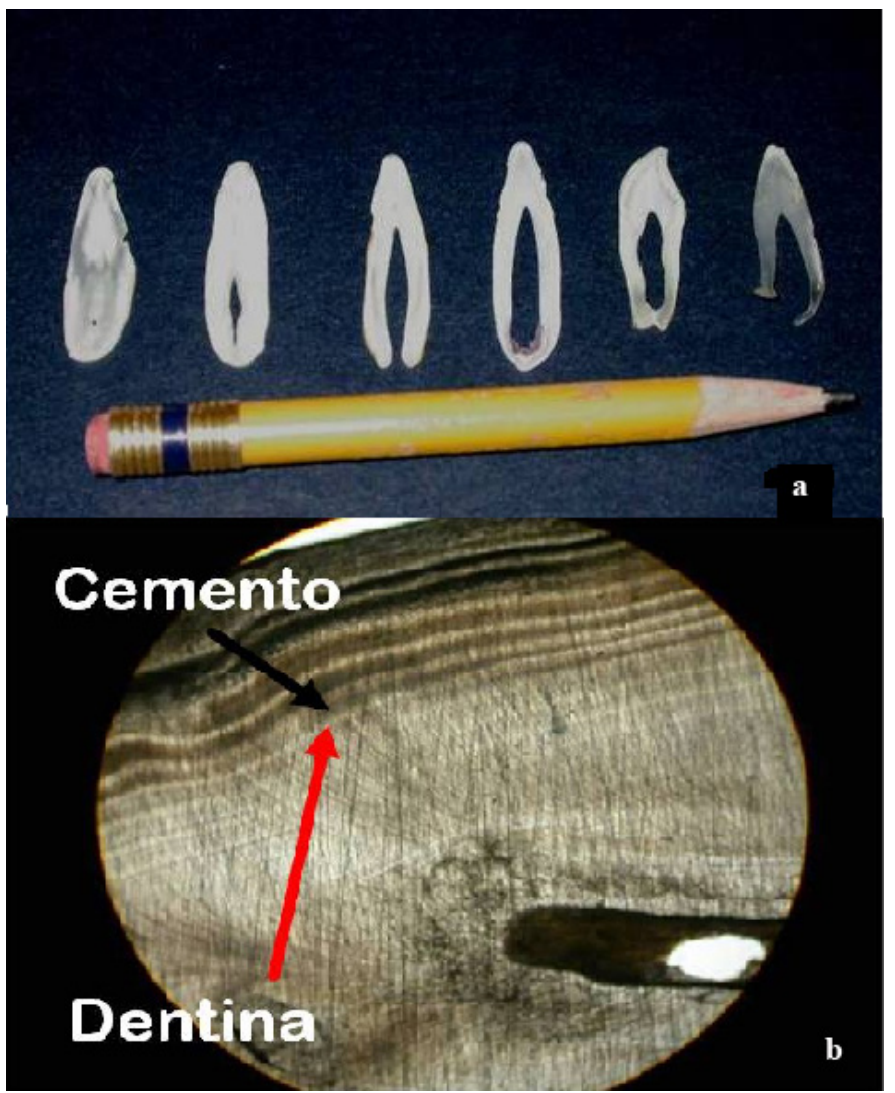
(40X).

Figura 1. Dientes de tonina preparados para su lectura en microscopio. (a) dientes laminados, (b) vista en microscopio óptico

anterior, se utilizó un flexómetro con graduación en $\mathrm{mm}$ y un par de maderas a fin de delimitar entre los puntos que habrían de medirse. De esta manera, al igual que en el caso de la relación de la longitud total con la edad, se elaboró la curva de crecimiento con los valores de edad y longitud total de los cráneos aplicando el modelo de crecimiento de von Bertalannfy. Así mismo, se realizó una prueba de " $\mathrm{t}$ " para comprobar qué tipo de relación existía entre la edad y la LCB.

\section{Resultados}

Se obtuvieron las edades y las medidas de longitud total de 42 toninas mexicanas y de 13 toninas cubanas, así como las longitudes condilobasales de 12 cráneos (Tabla 1). De los 42 delfines mexicanos, 18 fueron crías nacidas en cautiverio; tenían entre 10 meses (el menor) y casi cinco años de edad. Por lo tanto, se cuenta con un buen número de medidas en los primeros años de vida de estas toninas (Tabla 1). En cuanto a los sexos, en la muestra hay 13 machos (siete de Holbox y seis de Tabasco), 28 hembras ( 16 de Holbox, 10 de Tabasco y dos con padres de Holbox y Tabasco). Las toninas cubanas fueron ocho machos y cinco hembras. Las edades de las toninas mexicanas oscilaron entre " 0 " (recién nacidas) y 30 años, y en el caso de las toninas cubanas, entre los cuatro y los 19 años. Al nacer, el promedio de longitud total de las crías de progenitores provenientes de Holbox fue de $102.5 \pm 6.14 \mathrm{~cm}(n=4)$ en los machos, y $107 \pm 8.92 \mathrm{~cm}(n=4)$ en las hembras. Respecto de las crías de progenitores de Tabasco, fue de $110 \mathrm{~cm}(n=1)$ en machos y $107 \pm 3.21 \mathrm{~cm}(n=3)$ en hembras (Tabla 2). Al nacer, la longitud promedio delas toninas del sur del Golfo de México de ambos sexos fue de $106.11 \pm 6.22 \mathrm{~cm}(n=13)$. En los machos fue de $104 \pm$ $6.28 \mathrm{~cm}(n=5)$, y en las hembras, de $107 \pm 6.22 \mathrm{~cm}(n=8)$.

La longitud máxima teórica (Lmax) en las toninas mexicanas fue de $260 \mathrm{~cm}$ (Figura 2a). Por sexo, los machos presentaron una Lmax de $252 \mathrm{~cm}$, y las hembras de $258 \mathrm{~cm}$ (Figura 2b y 2c). 
Las toninas cubanas presentaron una Lmax de $249 \mathrm{~cm}$ (Figura 3a), $252 \mathrm{~cm}$ en machos, y $244 \mathrm{~cm}$ en las hembras (Figura 3b y $3 c$ ). Por ello, es notoria cierta diferencia en las longitudes asintóticas entre las toninas mexicanas y las cubanas, aunque estadísticamente no se encontraron diferencias significativas $(P<0.05)$.

En cuanto a las crías nacidas en cautiverio cuando se consideran ambos sexos en la curva de crecimiento su Lmax es de $234 \mathrm{~cm}$ (Figura 4a). Sin embargo, al separarlas por sexo, los machos presentan una Lmax de $236 \mathrm{~cm}$, dos centímetros menor a la Lmax de las hembras, que es de $238 \mathrm{~cm}$ (Figura $4 b$ y $4 c$ ).

\section{Discusión}

El modelo que describió el crecimiento de las toninas en este estudio fue el de L.v. Bertalanffy. Al comparar las curvas de crecimiento entre los sexos sin separar a los delfines por localidad, encontramos que ambas curvas son muy parecidas, y que las longitudes asintóticas difieren muy poco, a pesar de que en la curva de los machos los más longevos tienen 18 años, mientras que en las hembras la más longeva cuenta con 30 años. En ambos sexos, existen las clases de edad tempranas,

Tabla 1. Nombre, Sexo, Edad, Longitud Total (LT) y Longitud Condilobasal (LCB) de las toninas.

\begin{tabular}{|c|c|c|c|c|c|c|c|c|c|}
\hline \multicolumn{2}{|c|}{ NOMBRE } & \multirow{2}{*}{$\begin{array}{c}\text { EDAD } \\
18.0\end{array}$} & \multirow{2}{*}{$\begin{array}{c}\text { LT } \\
267.0\end{array}$} & \multirow[t]{2}{*}{ LCB } & \multicolumn{2}{|c|}{ NOMBRE } & \multirow{2}{*}{$\frac{\text { EDAD }}{6.0}$} & \multirow{2}{*}{$\begin{array}{c}\mathbf{L T} \\
220.0\end{array}$} & \multirow{2}{*}{$\begin{array}{c}\text { LCB } \\
444.0\end{array}$} \\
\hline ALUX & $\hat{\sigma}$ & & & & ZAMUA & $\hat{0}$ & & & \\
\hline HOLBOX & $\hat{\sigma}$ & 20.0 & 254.0 & 459.0 & $\mathrm{KI}^{\prime}$ & q & 0.5 & 145.0 & \\
\hline CHAME & $\hat{\sigma}$ & 11.0 & 239.0 & & D'XUNA & $\hat{\sigma}$ & 0.0 & 98.0 & \\
\hline IXCHEL & 우 & 18.0 & 252.0 & & KINAM & 우 & 1.9 & 214.5 & \\
\hline POLE & q & 13.0 & 234.0 & & XCARET & q & 4.9 & 224.0 & \\
\hline CHIQUILÁ & Á 우 & 18.0 & 240.0 & & KICHPAM & $\Lambda$ 우 & 4.9 & 228.0 & \\
\hline MELISSA & 우 & 13.0 & 250.0 & & ZAMA & 우 & 4.8 & 220.0 & \\
\hline MAYA & 우 & 16.0 & 261.0 & & SASTA & q & 4.7 & 219.0 & 450.0 \\
\hline FANNY & q & 16.0 & 254.5 & & SAS & 우 & 30.0 & 243.0 & \\
\hline ABRIL & q & 16.0 & 254.0 & & $\mathrm{KICH}$ & $\widehat{0}$ & 11.0 & 238.0 & \\
\hline XUNAH & 우 & 10.5 & 253.5 & & DZUL & $\hat{\sigma}$ & 14.0 & 238.0 & \\
\hline KAYNA & q & 15.5 & 242.0 & 476.0 & KAWAK & $\hat{0}$ & 12.0 & 228.5 & \\
\hline BAXAL & q & 3.5 & 225.0 & 451.0 & $\mathrm{AK}^{\prime} \mathrm{AB}$ & $\hat{\sigma}$ & 9.0 & 240.0 & \\
\hline XIB & 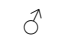 & 3.7 & 230.0 & 448.0 & NUSCA'A & A & 10.0 & 227.0 & \\
\hline WAYAK & $\widehat{0}$ & 2.2 & 221.0 & & IZAMAL & 우 & 11.0 & 228.5 & \\
\hline IXNUK & q & 2.1 & 221.0 & & TAPISH & q & 13.0 & 246.0 & \\
\hline $\mathrm{ICH}$ & $\hat{0}$ & 1.9 & 216.0 & & EK & $\sigma^{\pi}$ & 0.8 & 193.0 & \\
\hline KA'AN & q & 2.7 & 220.0 & & KANAY & 우 & 0.1 & 114.5 & \\
\hline HALKAB & q & 1.5 & 205.0 & & DZIC & $\sigma^{\pi}$ & 5 & & 440.0 \\
\hline KUX & q & 1.5 & 200.5 & & 1 & $\hat{0}$ & 18.0 & 265.0 & 473.0 \\
\hline TOS'HA & q & 1.9 & 202.0 & & 1 & $\hat{0}$ & 4.0 & 207.0 & 431.0 \\
\hline BAALAM & $\widehat{0}$ & 16.0 & 248.0 & 445.0 & 2 & ¿? & 3.0 & 200.0 & 424.0 \\
\hline CHAC & $\hat{\sigma}$ & 7.5 & 214.0 & & 3 & $\hat{0}$ & 20.0 & & 484.0 \\
\hline
\end{tabular}

Varamiento Tabasco. ${ }^{2}$ Varamiento Cd. del Carmen, Campeche. ${ }^{3}$ Varamiento Celestún, Campeche. 
representadas desde el nacimiento.

Cuando se comparan las curvas de crecimiento entre sexos de una misma localidad y entre estos con los de otra localidad, nos percatamos de que, entre los machos y las hembras de Holbox, la diferencia en la Lmax es mínima, y de que ambas curvas se comportan igual. Lo anterior debe tomarse con precaución, ya que la muestra de machos es muy pequeña y no se tiene representada la mayor parte de las clases de edad. Por ello, la diferencia en la longitud mínima es considerablemente mayor.

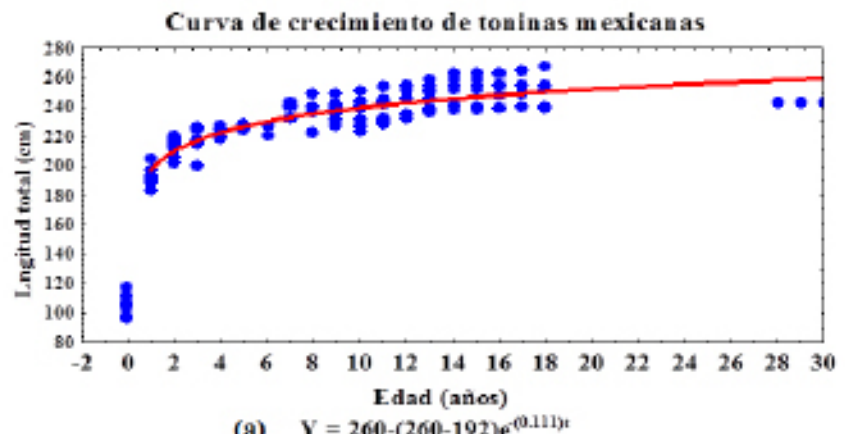

(a) $\quad \mathrm{Y}=260-(260-192) \mathrm{e}^{-(0.111) t}$

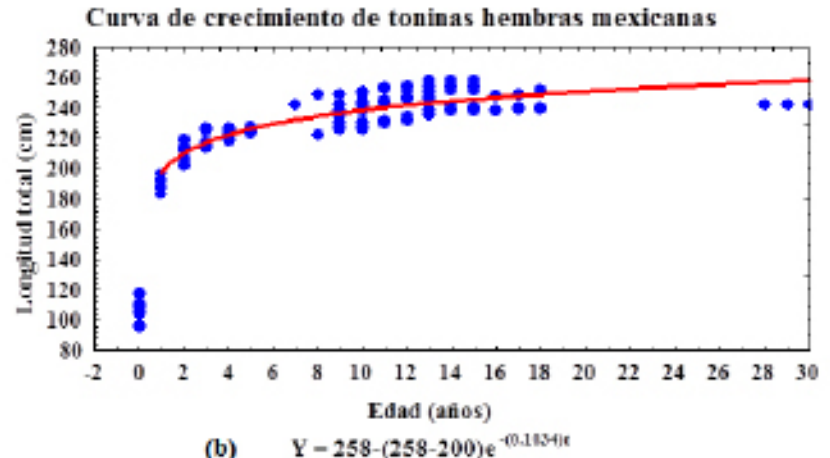

(b) $\quad \mathrm{Y}=258-(258-200) e^{-0.1134 i t}$

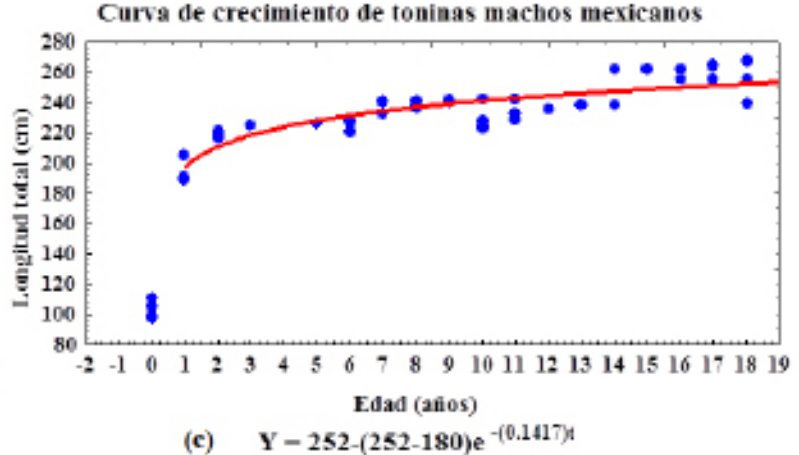

(c) $\quad \mathrm{Y}=252-(252-180) \mathrm{e}^{-(0.1417) t}$

Figura 2. Modelo general de crecimiento de toninas mexicanas (a), (b) hembras y (c) machos.

edad representadas también, en particular la curva de crecimiento de los machos de Tabasco, debe considerarse con mucho cuidado, puesto que es necesario ampliar la muestra para obtener resultados más precisos. Por su parte, la curva de crecimiento de las hembras de Tabasco resulta muy similar a la curva de las hembras de Holbox, que presentan Lmax casi iguales.

Las toninas cubanas, a pesar de que presentan un patrón de crecimiento aparentemente distinto al del patrón de los delfines mexicanos, y a que poseen una Lmax menor a la de los estos últimos, no manifiestan diferencias estadísticas significativas cuando se comparan las longitudes totales de edades similares entre ambos grupos de toninas $(P<0.05)$. Debe considerarse que no se cuenta con valores de longitudes totales de toninas menores a los cuatro años de edad. Lo anterior, sin duda, puede reflejarse en los resultados obtenidos en este análisis. Entre los machos y las hembras cubanas, no se hallaron diferencias estadísticas al comparar sus longitudes totales en diferentes edades $(P<0.05)$. Tampoco hubo diferencias al comparar clases de edad similares entre los machos de Holbox y los cubanos.

Read et al. (1993) obtuvieron longitudes totales de $250.1 \mathrm{~cm}$ en hembras de tonina, y de 263.4 cm en machos de la Florida. Fernández y Hohn (1998) determinaron longitudes asintóticas de $268 \mathrm{~cm}$ en machos, y de $246.7 \mathrm{~cm}$ en hembras de toninas de Texas. Cockroft y Ross (1989) 
Tabla 2. Longitud Total Promedio de las toninas al nacer y la localidad a la que pertenecen sus progenitores.

Localidad Promedio LT al nacer $\overparen{O}(\mathrm{~cm})$ Tamaño de muestra (n) Promedio LT al nacer $(\mathrm{cm})$ Tamaño de muestra (n)

\begin{tabular}{lllll}
\hline Holbox & $102.5 \pm 6.14$ & 4 & $107 \pm 8.92$ & 4 \\
Tabasco & 110 & 1 & $107 \pm 3.21$ & 3 \\
\hline Th= Longitud Total. & & & \\
\hline
\end{tabular}

LT= Longitud Total.

encontraron Lmax de $243 \mathrm{~cm}$ en machos, y de $238 \mathrm{~cm}$ en hembras de toninas de Sudáfrica. Los resultados obtenidos en este trabajo son más parecidos a los que se encontraron en Texas y en Florida, aunque los delfines mexicanos y los cubanos permanecen en un punto intermedio entre los norteamericanos y los sudafricanos. No obstante, la muestra de machos de México es reducida, y esto puede explicar parte de la diferencia encontrada. En consecuencia, es necesario incrementar la muestra y respaldar los datos anteriores con datos de otras investigaciones.

Por otra parte, debido a que Via Delphi ha tenido éxito en la reproducción de las toninas en cautiverio, los valores de longitud total y de las edades de las crías se conocen desde que estas nacen, lo que resulta difícil de obtener en la vida libre, dado que los animales pequeños aún se encuentran con sus madres y son dependientes de ellas.

En el nacimiento, la longitud total no presenta dimorfismo sexual $(P<0.05)$. Sin embargo, encontramos información interesante al percibir diferencias estadísticas significativas cuando se comparan los promedios de Lmax a distintas edades entre los sexos de los delfines de Holbox. En la muestra de los delfines de Tabasco, sólo existe un macho, y los valores de las hembras no son suficientes para realizar una comparación. En todos los casos, los machos fueron los que presentaron promedios más altos que las hembras, y se observa una tendencia a que los machos crezcan más

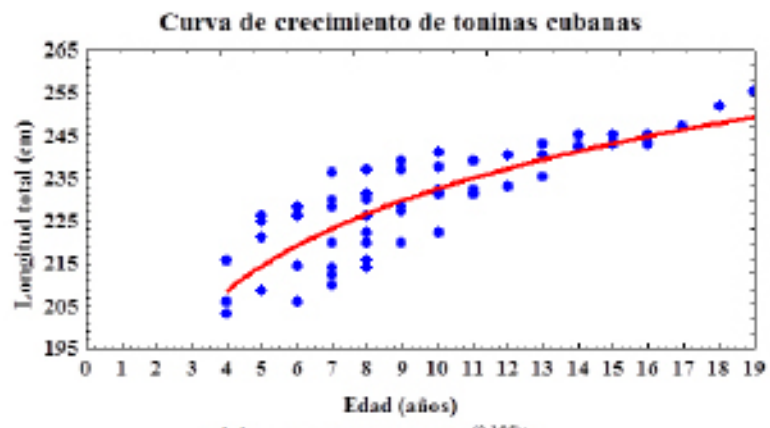

(a) $\quad Y-249-(249-157) e^{-(0.146) t}$
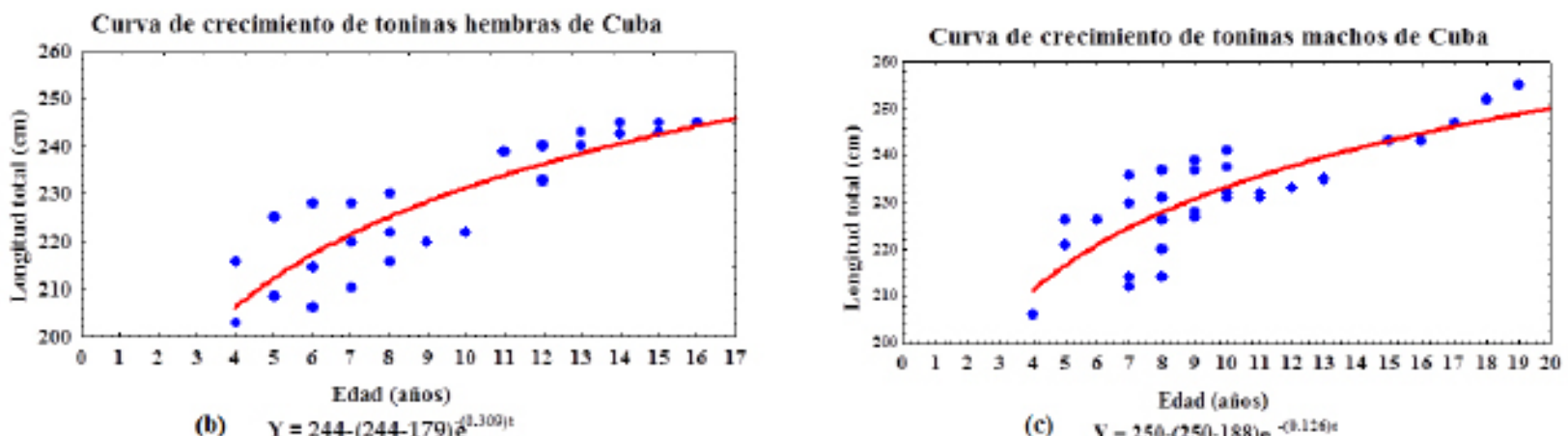

Figura 3. Modelo general de crecimiento de toninas cubanas (a), (b) toninas hembras y (c) toninas macho. 

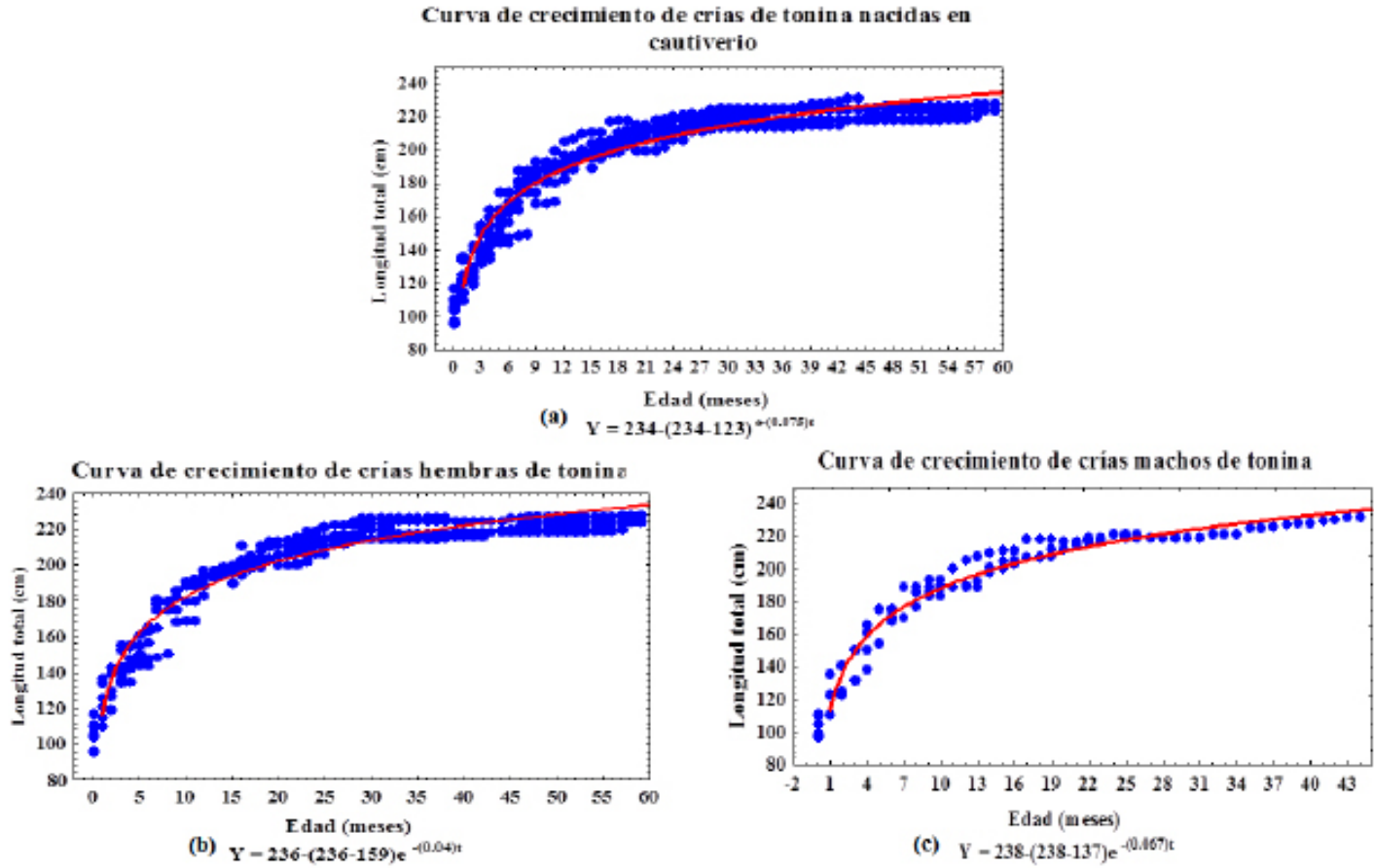

Figura 4. Modelo general de crecimiento de crías de tonina nacidas en cautiverio (a), (b) toninas hembra y (c) toninas macho.

rápido que las hembras. Lo anterior es muy importante, pues en los demás trabajos que se han realizado con toninas en vida libre, los resultados demuestran que las hembras son las que crecen más rápido que los machos, que alcanzan su longitud asintótica antes que estos. El fenómeno anterior se discute, ya que puede deberse a una adaptación para obtener un mayor éxito reproductivo (Read et al. 1993). Esto nos hace pensar que los delfines nacidos en cautiverio (al menos en Via Delphi) viven bajo condiciones constantes, con regímenes alimenticios controlados y sin problemas de depredación. También nos hace pensar que las diferencias sexuales en cuanto a tamaño -que en los animales de vida libre es evidente hasta la edad adulta-, en cautiverio se presentan a temprana edad.

Los valores de la longitud total cuando nacieron las toninas muestreadas son menores a los encontrados por Fernández y Hohn (1998) en toninas de Texas, con machos de $109.5 \pm 9.3 \mathrm{~cm}(n=$ $21)$, hembras $111.2 \pm 13.6 \mathrm{~cm}(n=5)$, y ambos sexos juntos de $109.5 \pm 9.3 \mathrm{~cm}(n=21)$. Los delfines de Sudáfrica presentaron valores por debajo de los promedios en las toninas de México, con 103 $\pm 7.6 \mathrm{~cm}(n=26)$. Sin embargo, estadísticamente no hay diferencias entre estos delfines.

Otro hallazgo importante con los resultados de las crías es que, al cumplir los dos años de edad y hasta aproximadamente los cinco años, el ritmo de crecimiento disminuye y se crea una meseta en la curva. No obstante, las crías de mayor edad incluidas en este trabajo tienen cinco años y será muy importante observar cómo se modifica su crecimiento en los siguientes años. Lo anterior podría significar, como en otras especies, que el crecimiento se desarrolle en dos o más fases. También es importante considerar que las hembras llegan a la madurez sexual entre los siete y los 10 años. Por lo tanto, probablemente la fase importante de crecimiento en longitud total se encuentre en los primeros cinco a siete años de vida y después invierten energía para la reproducción, ya no en el crecimiento. Por su parte, los machos que mantienen un ritmo de crecimiento constante, aunque también disminuye con la edad, al alcanzar la madurez sexual y la madurez física. Estos organismos invierten más energía en ganar masa corporal durante la madurez con fines de reproducción. 


\section{Curva de crecimiento Edad-Longitud Condilobasal}

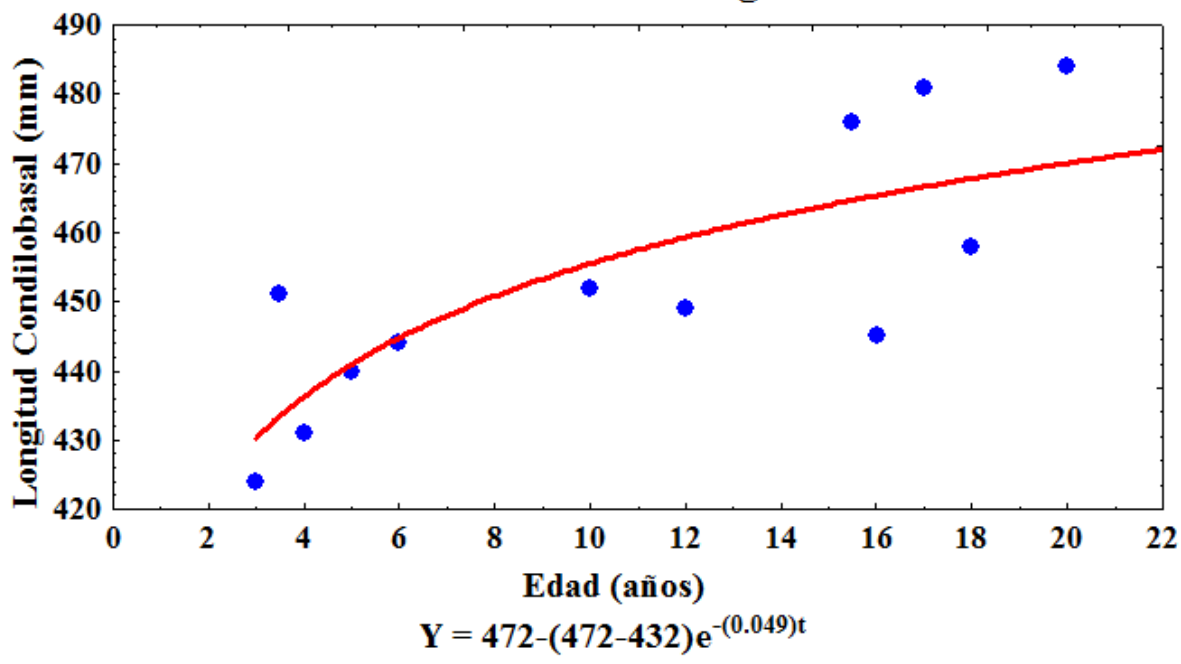

Figura 5. Modelo de crecimiento de cráneos de toninas del sur del Golfo de México.

Respecto de las medidas craneales, en la gráfica encontramos una dispersión alta de los valores, lo que puede deberse a que la muestra es muy pequeña. También es atribuible a que posiblemente se tengan valores que se encuentren en los límites máximo o mínimo de los intervalos en cada clase de edad. Así mismo, debe tenerse presente que se incluyen ambos sexos y que no se tienen representadas todas las clases de edad, al menos por un individuo. Por lo anterior, es necesario ampliar la muestra a fin de obtener mejores resultados (Figura 5).

Es de particular importancia seguir el desarrollo de estos animales y continuar recopilando información para obtener una mejor muestra, con resultados más confiables cuando las crías alcancen la madurez sexual y física.

\section{Conclusiones}

Los valores de Lmax de las toninas del sur del Golfo de México son parecidos a los de las toninas de Texas y Florida. No se encontraron diferencias estadísticas.

El modelo de crecimiento que mejor se ajustó a la muestra fue el de von Bertalanffy. No se encontraron diferencias en el crecimiento en las Lmax entre delfines adultos de las tres localidades ni entre los sexos.

En las crías de Holbox se encontraron diferencias estadísticas significativas entre los sexos en las longitudes totales en diferentes clases de edad. Los machos crecen más rápido que las hembras, o más bien son más grandes que ellas a la misma edad, en contraste con lo informado sobre toninas de la misma edad en vida libre.

El crecimiento de los cráneos no pudo establecerse debido al pequeño tamaño de muestra y a la amplia variación entre las medidas. Sin embargo, también son los primeros valores de crecimiento en toninas del sur del Golfo de México.

Finalmente, para contar con datos más confiables, es necesario ampliar el tamaño de la muestra y dar seguimiento a los que ya se tienen. 


\section{Agradecimientos}

Se agradece el apoyo y la confianza de los directivos de Grupo Via Delphi para realizar este trabajo. Se agradece al departamento de veterinaria, R. Torres y L. Ibarra, por su importante participación en la extracción de los dientes de los delfines. Se agradece a todos los entrenadores y manejadores por su valiosa participación en la obtención de las medidas de las toninas.

\section{Resumen}

Introducción: Este estudio se realizó para conocer las tasas de crecimiento de las toninas (Tursiops truncatus) mantenidas en resguardo por el Grupo Via Delphi de los estado de Quintana Roo y Tabasco en México, y de las toninas traídas de la República de Cuba.

Métodos: Se determinaron las edades de 43 delfines (algunos nacidos en cautiverio), se obtuvieron las longitudes totales de todos los individuos y las longitudes craneales de 12 toninas, así como el sexo de cada uno (17 machos y 26 hembras). Se verificó si existían diferencias estadísticas significativas entre los sexos y entre las localidades. De los 43 animales, 13 son crías de entre 10 meses de edad y cinco años.

Resultados: Para los machos y las hembras maduros sexualmente de la localidad de Quintana Roo, los promedios e intervalos de la longitud total y la edad de los individuos fueron los siguientes: los tres machos, de 18 años; longitud total promedio $=253.33 \mathrm{~cm} \pm 14 \mathrm{~cm}$ (intervalo $239-267 \mathrm{~cm}, \mathrm{n}=3$ ). Las hembras, todas mayores de 10 años; longitud total promedio $=250.6 \mathrm{~cm} \pm 7.5 \mathrm{~cm}$ (intervalo $239-261 \mathrm{~cm}, \mathrm{n}=8$ ). En la localidad de Tabasco, los valores fueron: todos los machos, mayores de nueve años; longitud total promedio $=236.13 \mathrm{~cm} \pm 5.2 \mathrm{~cm}$ (intervalo $238-240 \mathrm{~cm}, \mathrm{n}$ =4). Todas las hembras, mayores de los ocho años; longitud total promedio $=239.4 \mathrm{~cm} \pm 7.4 \mathrm{~cm}$ (intervalo $227-246$ $\mathrm{cm}, \mathrm{n}=5$ ). El total de toninas cubanas fue de siete; por tanto, los valores se consideraron aparte, con objeto de ser comparados con las toninas de la misma edad y sexo de las otras dos localidades.

Discusión: Los valores de Lmax de las toninas del sur del Golfo de México son parecidos a los de las toninas de Texas y Florida. No se encontraron diferencias estadísticas significativas. Tampoco se hallaron diferencias en el crecimiento de las Lmax entre delfines adultos ni entre localidades ni entre los sexos.

Palabras clave: Crecimiento; Cuba; edad; longitud total; México; toninas.

\section{Literatura citada}

Ballance, L. 1990. Residence patterns, group organization, and surfacing association of bottlenose dolphins in Kino Bay, Gulf of California, Mexico. Pp. 267-283 in The Bottlenose Dolphin (Leatherwood, S., y R. R. Reeves eds.). Academic Press. San Diego, EE. UU.

Bazúa-Durán, C., y A. Delgado-Estrella. 2014. Los tursiones, delfines de la laguna de Términos. Revista FOMIX-Campeche 19:20-26.

Berta, A., J. L. Sumich, y K. M. Kovacs. 2006. Marine Mammals: Evolutionary Biology. Academic Press. San Diego, EE. UU.

Cockroft, V. G., y G. J. B. Ross. 1989. Age, growth and reproduction of bottlenose dolphins, Tursiops truncatus, from the east coast of southern Africa. Fishery Bulletin 88:289-302.

Delgado-Estrella, A. 1997. Relación de las toninas Tursiops truncatus y las toninas moteadas Stenella frontalis con la actividad camaronera en la Sonda de Campeche, México. Anales del Instituto de Biología, Universidad Nacional Autónoma de México, Serie Zoología 68:317-338.

Fernández, S., y A. A. Hohn. 1998. Age, growth and calving season of bottlenose dolphins, Tursiops truncatus, off coastal Texas. Fishery Bulletin 96:357-365.

Нонn, А. А. 1980. Age determination and age related factors in the teeth of Western North Atlantic bottlenose dolphins. Scientific Reports of the Whales Research Institute 32:39-66.

Gallo-Reynoso, J.P . 1984. Interacción de calderones (Globicephala macrorhynchus), con la ballena de aleta (Balaenoptera physalus) y con tursiones (Tursiops truncatus gillii), (Mammalia:Cetacea). Anales del Instituto de Biología, Serie Zoología. UNAM. 55:331-333. 
Mead, J. G., y C. W. Potter. 1990. Natural history of bottlenose dolphins along the Central Atlantic Coast of the United States. Pp. 165-195 in The Bottlenose Dolphin (Leatherwood, S. y R. R. Reeves, eds.), Academic Press. San Diego, EE. UU.

Mrrick, A. 1991. Some new and potential uses of dental layers in studying delphinid populations. Pp. 251279 in Dolphin societies, discoveries and puzzle (Pryor K., y K. S. Norris eds.), University of California Press. Berkeley, EE. UU.

Read, A. J., R. S. Wells, A. A. Hohn, y M. D. Scott. 1993. Patterns of growth in wild bottlenose dolphins, Tursiops truncatus. Journal of Zoology 231:107-123.

Reeves, R. R., B. D. Smith, E. A. Crespo, y G. Notobartolo di Sciara (compiladores). 2003. Threats faced by cetaceans. Pp. 13-21 in Dolphins, Whales and Porpoises: 2002-2010 Conservation Action Plan for the World's Cetaceans. IUCN/SSC Cetacean Specialist Group. IUCN, Gland, Switzerland and Cambridge, UK.

WADE, P. R. 2002. Population dynamics. Pp. 974-979 in Encyclopedia of marine mammals (Perrin, W. F., B. Würsig, y J. G. M. Thewisen, eds.) Academic press. San Diego, EE. UU.

Summited: February 2, 2015

Review: March 12, 2015

Accepted: May 20, 2015

Associated editor: Juan Pablo Gallo 
400 THERYA Vol.6(2):389-400 